\title{
AVALIAÇÃo de ASPECTOS HIDRAÚlICOS E HIDROLÓgICOS DE SISTEMAS ALAGADOS CONSTRUÍDOS DE FLUXO SUBSUPERFICIAL
}

\section{EVALUATING HYDROLOGICAL AND HYDRAULIC ASPECTS IN SUBSURFACE-FLOW CONSTRUCTED WETLAND SYSTEMS}

\section{RESUMO}

Este trabalho teve-se como objetivo avaliar os aspectos hidráulicos e hidrológicos de quatro sistemas alagados construídos (SACs), cultivados com taboa (Typha sp.) e utilizados no tratamento de esgoto doméstico pré-decantado em tanque séptico. O sistema foi alimentado com uma taxa de aplicação hidráulica $(q)$ constante de 60, 47, 23 e 35 litro $\mathrm{m}^{-2} \mathrm{~d}^{-1}$, respectivamente, nos SACs 1, 2, 3 e 4, tendo recebido monitoramento e medição da condutividade hidráulica e evapotranspiração no sistema. Foram realizados balanços hídricos mensais, durante um período de seis meses. Os resultados evidenciaram que: a condutividade hidráulica no meio suporte é reduzida com o tempo de funcionamento do sistema; e o sistema de tratamento apresentou evapotranspiração média de $9,3 \mathrm{~mm} \mathrm{~d}^{-1}$, resultando num coeficiente da cultura $(\mathrm{Kc}) \mathrm{da}$ taboa com variação de 2,22 a 4,58.
\end{abstract}

MOZART DA SILVA BRASIL

Engenheiro Agrônomo. Doutor em Engenharia Agrícola pela Universidade Federal de Viçosa, Área de Recursos Hídricos e Ambientais. Professor da Escola Agrotécnica Federal de Colorado do Oeste-RO

\section{Antonio TeIXEIRA DE MATOS}

Engenheiro Agrícola. Doutor em Solos e Nutrição de Plantas pela Universidade Federal de Viçosa. Professor Associado I do Departamento de Engenharia Agrícola e Ambiental da UFV, na Área de Recursos Hídricos e Ambientais

Recebido: 29/07/07 Aceito: 29/07/08
PALAVRAS-CHAVE: Esgoto doméstico, águas residuárias, evapotranspiração, coeficiente de cultura, condutividade hidráulica, sistemas alagados construídos.

\begin{abstract}
This work was conducted to evaluate both hydrological and hydraulic aspects in four constructed wetland systems (SACs), that were cropped with Typha sp. and used in the treatment of the domestic wastewater presettled in septic tank. The system was fed a constant water application ratio (q) of 60, 47, 23 and 35 liter $m^{-2} d^{-1}$ in SACs 1, 2, 3 and 4, respectively, besides being monitored. The hydraulic conductivity and evapotranspiration were also measured. Water balances were monthly accomplished during six-months period. The results evidenced the hydraulic conductivity in the supportive medium to be reduced during the operating time of the system, as well as the treatment system showed an average evapotranspiration of $9.3 \mathrm{~mm} \mathrm{~d}^{-1}$, as resulting into a cropping coefficient $(K c)$ of the Typha sp. ranging from 2.22 to 4.58 .
\end{abstract}

KEYWORDS: Domestic wastewater, wastewater, evapotranspiration, crop coefficient, hydraulic conductivity, constructed wetland systems.

\section{INTRODUÇÃO}

O processo de tratamento de água residuária em sistemas alagados construídos (sistemas "Wetlands"), cultivados com plantas macrófitas, caracterizam-se como sistemas robustos e de baixos custos e simplicidade de operação e manutenção. Tais aspectos os tornam ideais para aplicação em regiōes carentes de saneamento básico, adequando-se perfeitamente às condições de países de clima tropical, que possuam áreas disponíveis à sua implantação, como é o caso do Brasil.

Os referidos sistemas de tratamento de águas residuárias são adequados para se fazer o tratamento secundário e terciário de despejos domésticos (Brasil,
2007) e industriais, além de produzir efluentes com excelente qualidade para o reuso na agricultura (Brasil, 2005), desde que se observem os critérios de segurança de saúde pública. Todavia, o seu caráter extensivo sobre a superfície assemelha-o a ecossistemas naturais, estando sujeito a perdas de águas por evapotranspiração, o que pode os tornar desinteressantes em regiões áridas ou semi-áridas, quando o objetivo for o reuso do efluente na agricultura. Em condições experimentais na costa do Marrocos, registrou-se evapotranspiração de $38 \mathrm{~mm} \mathrm{~d}^{-1}$ em Sistema Alagado Construído (SAC) cultivado com Arundo danox (Cadelli et al, 2004) e na Malásia, em sistemas alagados, com fluxo subsuperficial, cultivados com
Typha angustifólia, registrou-se evapotranspiração de $32,9 \mathrm{~mm} \mathrm{~d}^{-1}$.

A eficiência do sistema pode ser influenciada pelo ciclo hidrológico e, segundo Davis (1995), as precipitações e as perdas de água por evapotranspiração devem ser consideradas no dimensionamento do mesmo. As precipitaçôes podem provocar elevação temporária do nível da água, alterando o comportamento hidráulico e podendo causar escoamento superficial no sistema, e, com isso, proporcionando redução na eficiência do tratamento. A ocorrência da evapotranspiração reduz, por sua vez, o nível da água no tanque e, por conseqüência, proporciona concentração dos poluentes presentes na água residuária em tratamento, possibilitando subesti- 
mativa da eficiência de remoção pelos reatores e, em certos casos, podendo provocar efeitos tóxicos à vegetação.

$\mathrm{O}$ escoamento superficial indesejado no SAC pode, também, surgir em consequiência do aumento na perda de carga no substrato, em decorrência do acúmulo de lodo, ao longo do tempo de operação do sistema. A variação na condutividade hidráulica no meio suporte ocorre tanto em função do tempo como em função da posição no tanque. No entanto, a condutividade hidráulica no meio suporte é difícil de ser medida no sistema, porque é influenciada por fatores como a ocorrência de fluxo preferencial, curto circuito e obstrução da porosidade (em conseqüência de variações de crescimento e degradação de raízes e acumulação/degradação de resíduos sólidos no SAC). A redução no valor da condutividade hidráulica ocorre, em maior magnitude, no primeiro terço dos leitos. Como o nível do líquido nos leitos deve permanecer em torno de $10 \mathrm{~cm}$ abaixo da superfície do meio suporte, para dimensionamento do sistema, sugere-se usar valores conservativos de condutividade hidráulica, isto é, de $1 \%$ e $10 \%$ do valor obtido em meio suporte limpo, respectivamente, para estimar a perda de carga nos primeiros $30 \%$ e nos últimos $70 \%$ da extensão dos leitos (U.S. EPA, 2000).

A evapotranspiração em sistemas alagados naturais pode estar relacionada ao clima e às características das águas de subsolo e de superfície, como também, à fisiologia das plantas. A evaporação e a transpiração são influenciadas pelas condições meteorológicas, tais como radiação solar ou temperatura da superfície e por fatores como a umidade relativa do ar, velocidade do vento (Sánchez-Carrillo et al, 2001).

Com a realização deste trabalho, objetivou-se medir a condutividade hidráulica e a evapotranspiração e calcular o coeficiente de cultura $(\mathrm{Kc}) \mathrm{da}$ taboa, cultivada em Sistemas Alagados Construídos, usado no tratamento de esgoto doméstico.

\section{MATERIAL E MÉTODO}

O experimento foi conduzido na Área Experimental de Tratamento de Resíduos (AETR) do Departamento de Engenharia Agrícola da Universidade Federal de Viçosa - DEA/UFV, em Viçosa, Brasil. Quatro Sistemas Alagados Construídos (SAC) foram instalados em paralelo, nas dimensōes e declividades apresentadas nas Figuras 1 e 2 , respectivamente, para tratamento secundário/terciário de esgoto doméstico. Como meio suporte, utilizou-se brita \# 0 (diâmetro $-\mathrm{D}_{60}=7,0 \mathrm{~mm}$, $\mathrm{Cu} \mathrm{D}_{60} / \mathrm{D}_{10}=1,6$, volume de vazios de $48,4 \%$ e condutividade hidráulica saturada $\mathrm{K}_{\mathrm{s} 20}=7.970 \mathrm{~m} \mathrm{~d}^{-1}$ ). A espécie vegetal emergente cultivada nos SACs foi a taboa (Typha sp).

O volume de vazios da brita foi quantificado em laboratório, empregando-se proveta graduada de 1 litro de volume e água destilada. A condutividade hidráulica saturada $\left(\mathrm{K}_{\mathrm{s}}\right)$ foi determinada baseando-se nos princípios da lei de Darcy (metodologia apresentada por Ferreira, 2002) e utilizando-se permeâmetro horizontal, constituído por uma coluna de PVC de $0,50 \mathrm{~m}$ de comprimento e $0,075 \mathrm{~m}$ de diâmetro. Para caracterização das condiçôes térmicas do escoamento, monitorou-se a temperatura da água utilizada por meio de um termômetro analógico de mercúrio.
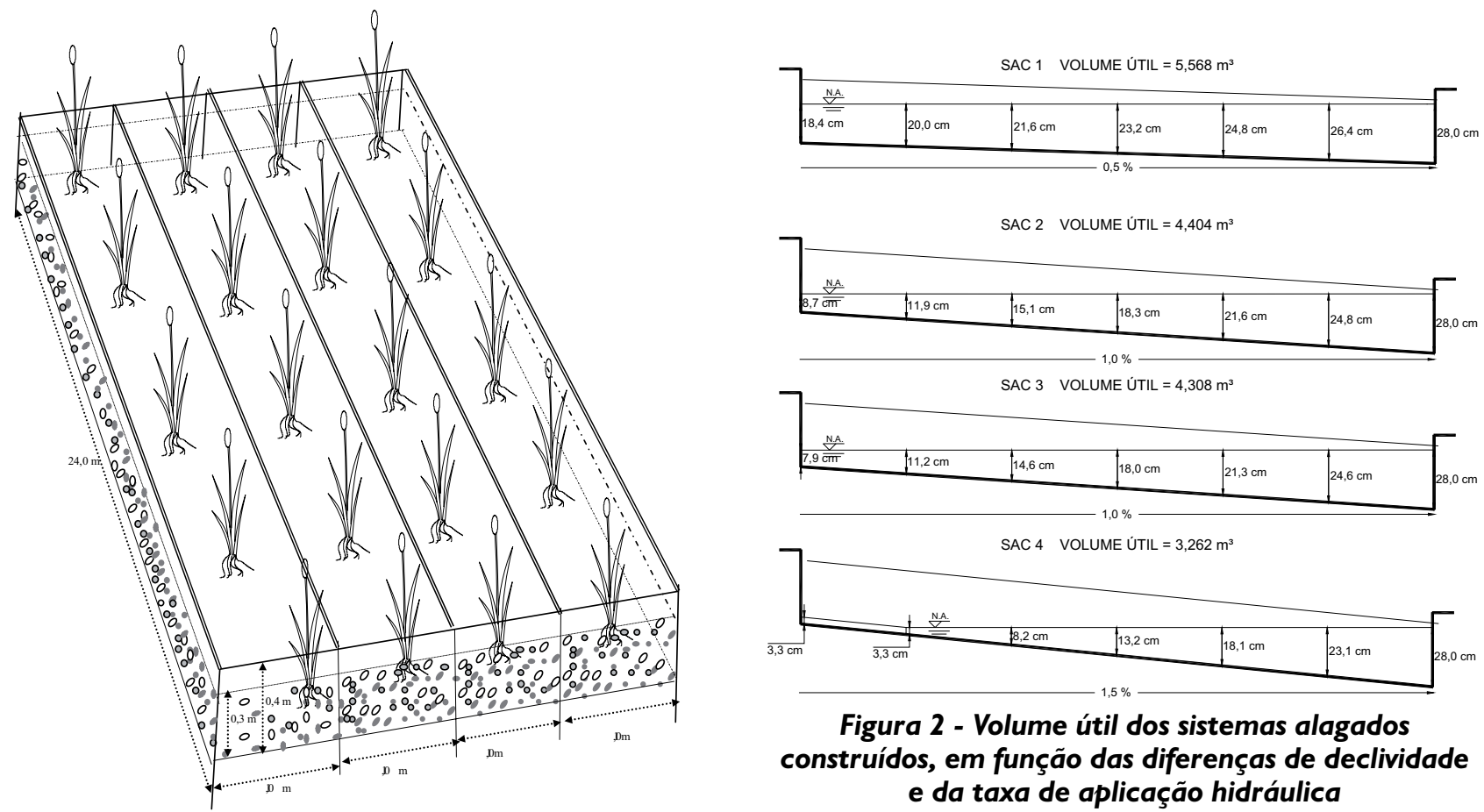

Figura 2 - Volume útil dos sistemas alagados construídos, em função das diferenças de declividade e da taxa de aplicação hidráulica

Figura I - Diagrama esquemático dos quatro sistemas alagados construídos 
Ao longo dos SACs, foram instalados, de 4 em $4 \mathrm{~m}$, distribuídos no sentido longitudinal, tubos de PVC com $40 \mathrm{~mm}$ de diâmetro, cravados verticalmente no meio suporte, para possibilitar coleta de amostras do líquido em tratamento nos SACs. Os quatro SACs foram alimentados com efluente doméstico, proveniente de tanque séptico, sob taxa de aplicação hidráulica média $(q)$ e tempos de residência hidráulica $(t)$ constantes ao longo do tempo de experimentação. O valor de $t$ foi, entretanto, diferente entre os SACs, conforme apresentado no Tabela 1 .

Os valores de vazão média foram obtidos com o cálculo das vazōes médias nos leitos $\left[\left(Q_{\text {afuente }}+Q_{\text {efluente }}\right) / 2\right] \mathrm{e}$ da seção média entre dois pontos de coletas subseqüentes (Figura 2). Para o cálculo das taxas de aplicação hidráulica superficial $\left(q_{a}\right)$ médias, utilizou-se a vazão média através dos leitos e a superfície do SAC, enquanto, para as taxas de aplicação hidráulica volumétrica $(q)$ utilizou-se o volume de vazios do SAC (volume útil $\mathrm{x}$ macroporosidade do meio suporte). As $q$ foram fixas para cada SAC, conforme pode está apresentado na Tabela 1 , uma vez que o volume útil de cada SAC, determinado no início da operação do sistema, era diferente (Figura 2).

As perdas de água por evapotranspiração foram calculadas para correção diária da vazão afluente, bem como para garantir vazão média definida por tratamento. A quantificação dessa perda foi obtida da diferença entre as vazões afluentes e efluentes, medidas no horário do dia em que essa perda de água representava o valor médio. Para encontrar o horário em que ocorria a evapotranspiração média, que correspondeu ao período de 9 às 11 horas, foram feitos balanços hídricos mensais, durante seis meses, com medidas de vazôes, em intervalos de 60 minutos, durante 24 horas do dia. O horário de ocorrência da evapotranspiração média era utilizado para medição das vazões de água residuária no sistema até a realização do balanço seguinte.

Os valores de evapotranspiração de referência $\left(\mathrm{ET}_{0}\right)$, foram obtidos a partir de dados meteorológicos medidos em estação meteorológica instalada na Área Experimental, utilizando-se a equação de Penman-Monteith, conforme Allen et al, (1998).

A quantificação das vazões afluentes e efluentes foi feita por meio de mediçôes diretas, no tempo de 60 segundos, utilizando-se de proveta graduada de volume de 1 litro.

Os valores de condutividade hidráulica saturada $(K)$ nos SACs em operação foram obtidos pelo método direto, aplicando-os efluente primário do tanque séptico, em que aumentouse a vazão afluente, controlando-a, de modo a elevar o nível da água residuária dentro do meio suporte, mantendo-o numa altura de $28 \mathrm{~cm}$ em todos os pontos de amostragem ao longo dos SACs, fazendo coincidir o gradiente da linha d'água com o gradiente do fundo. Após a estabilização do nível e das vazôes afluente e efluente, utilizou-se o valor de vazão efluente e a equação de Darcy, em que $K_{s}=\left[Q / A_{s} S\right]$, em que, $A$ é área da seção transversal ocupada pela água residuária no leito $\left(\mathrm{m}^{2}\right) ; S$ é gradiente hidráulico $\left(\mathrm{m} \mathrm{m}^{-1}\right)$ e $Q$ é vazão efluente $\left(\mathrm{m}^{3} \mathrm{~d}^{-1}\right)$.

O sistema foi avaliado por 9 meses e 15 dias, sendo alimentado do seguinte modo: 20 dias com esgoto doméstico, apenas dessarenado; 100 dias com esgoto doméstico dessarenado e decantado; e 165 dias com efluente de doméstico de tanque séptico.

\section{RESULTADOS E DISCUSSÃO}

\section{Aspectos hidráulicos de operação do sistema}

Aos 20 dias de operação, os SACs começaram a exibir escoamento superficial, com a água aflorando acima do nível do leito. O escoamento manifestou-se, primeiramente, no SAC de menor declividade $(0,5 \%)$, seguindo-se para os demais SACs, na ordem do de menor para o de maior declividade. Esse escoamento ocorreu, inicialmente, em pequena magnitude, tendo aumentado rapidamente com o passar do tempo de operação do sistema, sendo que o fenômeno localizou-se nos primeiros $2 \mathrm{~m}$, a partir da zona de entrada do afluente, em todos os SACs.

Este comportamento é decorrente das características físicas do afluente aos SACs, que consistia numa água residuária doméstica submetida simplesmente ao tratamento preliminar (gradeamento e desarenação). Nessa situação, o afluente apresentava concentração média de $15 \mathrm{~mL} \mathrm{~L}^{-1}$ de sólidos sedimentáveis, o que proporcionou rápido acúmulo de sólidos nos interstícios do meio suporte do início dos SACs e, consequentemente, redução no valor da condutividade hidráulica, nesta zona, fato que, de forma similar, também foi observado por Tanner \& Sukias (1995), induzindo a ocorrência de escoamento superficial nos leitos. Após esta constatação, passou-se a alimentar os SACs, como forma de avaliação, com esgoto doméstico submetido ao mesmo tratamento preliminar, só que

Tabela I - Valores médios de vazão $\left[\left(Q_{\text {afuente }}+Q_{\text {effuente }}\right) / 2\right]$, de tempo de residência hidráulica $(t)$ e de taxa de aplicação hidráulica (q) utilizadas nos leitos dos SACs

\begin{tabular}{cccccccc}
\hline SAC & $\begin{array}{c}\text { Vazão } \\
\text { média } \\
\left(\mathrm{m}^{3} \mathrm{~d}^{-1}\right)\end{array}$ & $\begin{array}{c}\mathrm{t} \\
(\mathrm{dia})\end{array}$ & $\begin{array}{c}\text { Área } \\
\left(\mathrm{m}^{2}\right)\end{array}$ & $\begin{array}{c}\text { V. útil } \\
\left(\mathrm{m}^{3}\right)\end{array}$ & $\begin{array}{c}\text { V. útil } \\
\mathrm{x} \\
\text { Porosidade } \\
\left(\mathrm{m}^{3}\right)\end{array}$ & $\begin{array}{c}\mathrm{q}_{\mathrm{a}}^{*} \\
\left(\mathrm{~L} \mathrm{~m}^{-2} \mathrm{~d}^{-1}\right)\end{array}$ & $\begin{array}{c}\mathrm{q}_{\mathrm{v}}^{* *} \\
\left(\mathrm{~m}^{3} \mathrm{~m}^{-3} \mathrm{~d}^{-1}\right)\end{array}$ \\
\hline 1 & 1,437 & 1,876 & 24,0 & 5,568 & 2,695 & 59,8 & 0,533 \\
2 & 1,135 & 1,876 & 24,0 & 4,404 & 2,131 & 47,3 & 0,533 \\
3 & 0,556 & 3,752 & 24,0 & 4,308 & 2,085 & 23,2 & 0,258 \\
4 & 0,840 & 1,876 & 24,0 & 3,262 & 1,579 & 35,0 & 0,533 \\
\hline
\end{tabular}


seguido de decantação. Este procedimento reverteu o quadro de ocorrência de escoamento superficial nos leitos, já que, após 10 dias de alteração na forma de tratamento da água residuária, o sistema passou a operar com escoamento exclusivamente subsuperficial. Em vista disso, decidiu-se operar o sistema com a aplicação do efluente de tanque séptico, especialmente construído para este fim, produzindo um efluente com características apresentadas na Tabela 2. Estes resultados evidenciam que SACs com características similares às investigadas não são viáveis para o tratamento de esgoto doméstico bruto, em vista da grande quantidade de sólidos nele presente, o que pode concorrer para rápida colmatação do espaço poroso do meio suporte, podendo vir a ocasionar o escoamento superficial nos leitos, com conseqüente redução na eficiência do sistema e o risco da exalação de maus odores.

Os resultados desta pesquisa corroboram as sugestóes de Reed et al (1995), Davis (1995), U.S. EPA (2000) e ITRC (2003), que desaconselham o tratamento de esgoto doméstico bruto em Sistemas Alagados Construídos.
Após a operacionalização de aplicação de efluente de tanque séptico nos SACs, mesmo quando ocorreram eventos de precipitação durante o monitoramento do sistema, não foi observado escoamento superficial nos leitos.

De acordo com os dados apresentados na Tabela 3, os valores de condutividade hidráulica, em condiçôes de saturação $(K)$ do meio suporte dos SACs, evidenciaram que, no início da avaliação do sistema, aos quatro meses após o plantio da vegetação, o valor de $K$ medido no meio suporte era muito superior ao do $K$ medido na brita "limpa", sem cultivo, que foi de $7.970 \mathrm{~m} \mathrm{~d}^{-1}$, sob uma temperatura de $20^{\circ} \mathrm{C}$. No final do período de monitoramento do sistema, o valor de $K$ já se apresentava bastante reduzido, mas ainda ligeiramente superior ao da brita "limpa", salientando que essa medida tenha sido feita com a água residuária apresentando temperatura de $22^{\circ} \mathrm{C}$, ou seja, superior aos $20^{\circ} \mathrm{C}$ da determinação inicial. Estes valores de condutividade hidráulica $(K)$ estão no intervalo de valores reportados por U.S. EPA (2000), para brita com diâmetro variando de
5 a $10 \mathrm{~mm}$, ou seja, $34.000 \mathrm{~m} \mathrm{~d}^{-1}$ para brita "limpa" e de $900 \mathrm{md}^{-1}$ para os primeiros $20 \mathrm{~m}$ do SAC em operação por 2 anos.

Em virtude dos autos valores de condutividade hidráulica e vazão nos SACs 3 e 4, aos quatro meses após o plantio, não foi possível medir o valor de $K$ nesses leitos.

O incremento no valor da condutividade hidráulica do meio suporte, em relação à brita "limpa", observado no início da avaliação dos SACs, pode ser creditado à abertura de novos caminhos para escoamento da água residuária, decorrente do desenvolvimento das raízes das plantas, como ocorre com meio suporte de solo, tal como reportado por Brix (1997). Esse comportamento pode ser presumido pela constatação do soerguimento do material do meio suporte, após o estabelecimento da vegetação. Também, pode-se creditar o incremento da condutividade hidráulica à diminuição da rugosidade da brita, em virtude do desenvolvimento do biofilme em sua superfície.

A redução observada no valor da condutividade hidráulica nos SACs, ao

Tabela 2 - Características físicas, químicas, bioquímicas e biológicas, médias, do efluente primário do tanque séptico

\begin{tabular}{ccc}
\hline Variáveis & Unidade & Valor \\
\hline $\mathrm{DQO}$ & $\mathrm{mg} \mathrm{L}^{-1}$ & 416 \\
$\mathrm{DBO}_{5}$ & $\mathrm{mg} \mathrm{L}^{-1}$ & 203 \\
Sólidos sedimentáveis & $\mathrm{mL} \mathrm{L}^{-1}$ & 1 \\
Sólidos suspensos totais & $\mathrm{mg} \mathrm{L}^{-1}$ & 75 \\
$\mathrm{pH}$ & - & 6,6 \\
Turbidez & $\mathrm{UNT}$ & 60 \\
Coliformes totais & $\mathrm{NMP} / 100 \mathrm{~mL}$ & $8,72 \mathrm{E}+07$ \\
\hline
\end{tabular}

Tabela 3 - Valores de condutividade hidráulica saturada, $K_{s}\left(\mathrm{~m} \mathrm{~d}^{-1}\right)$ e perda de carga $(m)$, determinadas durante o período de monitoramento dos sistemas alagados construídos

\begin{tabular}{|c|c|c|c|c|}
\hline \multirow[t]{2}{*}{ SAC } & \multicolumn{3}{|c|}{ Condutividade hidráulica } & \multirow{2}{*}{$\begin{array}{l}\text { Perda de carga } \\
\text { Entre início e final da avaliação } \\
\text { (relativa à vazão de operação) }\end{array}$} \\
\hline & $\begin{array}{c}\text { Brita "limpa" } \\
\mathrm{T}=20^{\circ} \mathrm{C}\end{array}$ & $\begin{array}{c}\text { Início da } \\
\text { avaliação } \\
\text { (4 meses após o } \\
\text { plantio) } \\
\mathrm{T}=20^{\circ} \mathrm{C}\end{array}$ & $\begin{array}{l}\text { Final da avaliação } \\
\left(5,5 \text { meses após } 1^{\text {a }}\right. \\
\text { avaliação }) \\
T=20^{\circ} \mathrm{C}\end{array}$ & \\
\hline 1 & 7.970 & 15.034 & 8.392 & 0,006 \\
\hline 2 & 7.970 & 15.813 & 9.099 & 0,005 \\
\hline 3 & 7.970 & - & 9.202 & - \\
\hline 4 & 7.970 & - & 9.885 & - \\
\hline Média & & 15.423 & 9.144 & 0,005 \\
\hline
\end{tabular}


final do período de monitoramento, em comparação aos valores obtidos no início da experimentação, foi semelhante àqueles observados por Sanford et al (1995), ao longo do tempo de operação do mesmo tipo de sistema, em Ithaca, USA. Este comportamento pode ser esperado, pois, durante o tratamento da água residuária ocorre, dentro dos SACs, o desenvolvimento do biofilme, a formação de precipitados e a retenção de sólidos suspensos do efluente, que contribuem para obstrução do espaço poroso e, conseqüentemente, diminuição na condutividade hidráulica no meio suporte.

$\mathrm{Na}$ Tabela 3, é possível verificar, ainda, que valores de $K_{s}$ expressam tendência de ligeiro aumento na ordem inversa do valor de volume útil dos SACs (Figura 2). Essa tendência pode ser justificada pelo fato de os SACs de menor volume útil disporem de maior volume de meio-suporte sem resíduos ou biofilme e, por conseguinte, maior profundidade de brita "limpa" acima da profundidade molhada, o que contribuiu para o incremento de $K$ nesses SACs, visto que todo a camada de $28 \mathrm{~cm}$ acima da camada impermeável foi considerada no cálculo do fluxo, utilizado na quantificação da condutividade hidráulica.

\section{Evapotranspição}

$\mathrm{Na}$ Tabela 4, estão apresentados os resultados de evapotranspiração da cultura (ETc), evapotranspiração de referência $\left(\mathrm{ET}_{0}\right)$ e coeficiente de cultura $(\mathrm{Kc})$, podendo-se observar tendência de obtenção de maiores valores de ETc nos SACs 1 e 4. Isto ocorreu, provavelmente, em razão de terem estes SACs apresentado maior número de brotos de taboa por volume de meio suporte, bem como por serem, entre os SACs avaliados, aqueles que receberam maior insolação diária, em virtude de estarem posicionados nas bordas da área experimental, perpendicularmente à direção do sol, sendo que o SAC 1 recebia maior incidência de radiação no período da manhã e o SAC 4 no período da tarde.

A tendência de aumento no valor da $\mathrm{ET}_{0}$ da primeira até à terceira amostragem justifica-se pelo fato de que, nesse período, as condições climáticas mudaram, passando da estação do inverno para a da primavera. $\mathrm{Na}$ quarta amostragem, já em condiçōes climáticas de verão, a $\mathrm{ET}_{0}$ apresentou menor magnitude, tendo em vista que o período era chuvoso, conforme pode ser observado na Figura 3.
O valor máximo de $14 \mathrm{~mm} \mathrm{~d}^{-1} \mathrm{de}$ evapotranspiração, obtido pela espécie Typha sp., foi inferior ao valor obtido por Lim et al. (2001), em sistemas alagados, com fluxo subsuperficial, cultivados com Typha angustifólia, sob clima tropical, na Malásia, que variou de 18,2 a $32,9 \mathrm{~mm} \mathrm{~d}^{-1}$.

A porcentagem de água perdida em relação à lâmina afluente aplicada variou de $11 \%$ a $27 \%$, mostrando-se inferior à perda de $30 \%$, observada, na estação da primavera, em Pádua, Itália (Borin et al, 2004) e de 50\%, sob temperaturas de 30 a $34^{\circ} \mathrm{C}$, em Campina Grande, Brasil (Sousa et al, 2004), em Sistemas Alagados Construídos.

$\mathrm{O}$ valor do Kc na primeira amostragem foi de 3,00, quando os brotos da taboa estavam com altura em torno de $1,3 \mathrm{~m}$. Na segunda amostragem, o valor do Kc foi de 4,58, quando as plantas encontravam-se em pleno desenvolvimento fisiológico, com altura em torno de 2,0 m, e já haviam iniciado o florescimento. Na terceira amostragem, período coincidente com o início da senescência das plantas, o valor do Kc foi de 3,28. Com base nos resultados obtidos, verificou-se que o valor do Kc depende, em SACs, tal como ocorre com plantas de cultivo convencional, do

Tabela 4 - Valores de ETc, ET 0 e Kc obtidos durante o período de monitoramento do sistema

\begin{tabular}{cccccccc}
\hline Data & \multicolumn{3}{c}{ ETc $\left(\mathrm{mm} \mathrm{d}^{-1}\right)$} & & \multicolumn{2}{c}{ ET $_{0}$} \\
& SAC 1 & SAC 2 & SAC 3 & SAC 4 & Média & $\left(\mathrm{mm} \mathrm{d}^{-1}\right)$ & Kc $^{2}$ \\
\hline $20 / 08 / 04$ & 8,40 & 6,81 & 6,42 & 7,47 & 7,27 & 2,42 & 3,00 \\
$14 / 09 / 04$ & 18,73 & 11,65 & 11,83 & 13,91 & 14,03 & 3,06 & 4,58 \\
$23 / 11 / 04$ & 12,70 & 7,40 & 9,33 & 14,61 & 11,01 & 3,36 & 3,28 \\
$14 / 01 / 05$ & 6,45 & 5,58 & 3,61 & 4,04 & 4,92 & 2,22 & 2,22 \\
Média & - & - & - & - & 9,30 & - & 3,27 \\
\hline
\end{tabular}

Kc $=$ ETc médio/ETo

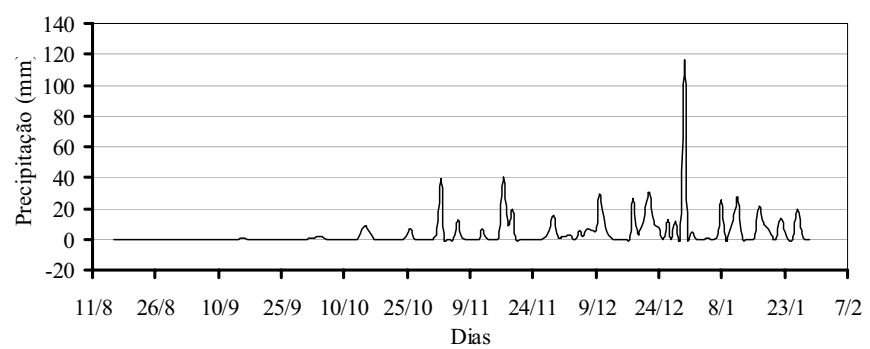

Figura 3 - Valores de precipitação registrados no período de monitoramento do experimento 
estádio fenológico das plantas. $\mathrm{Na}$ quarta amostragem, as plantas tinham retomado o rebrotamento, após efetuado o seu corte, e estavam com altura em torno de 1,2 $\mathrm{m}$, apresentando, por sua vez, um valor de Kc de 2,22. Este decréscimo no valor do Kc pode ser atribuído à menor área foliar, o que proporcionou redução na transpiração das plantas. Considerando que a taboa é uma espécie vegetal de ciclo perene, pode-se adotar um valor médio de Kc de 3,27.

\section{CONCLUSÕES}

Para as condições experimentais deste trabalho e o período de observação, os resultados obtidos indicaram que:

- Caso os SACs sejam aplicados no tratamento de efluente de tanque séptico, estes são passíveis de operar apresentando apenas fluxo subsuperficial, ou seja, sem afloramento na superfície do leito;

- A condutividade hidráulica do meio suporte diminuiu com o tempo de 285 dias de operação do sistema, embora tenha se mantido acima da obtida em brita "limpa";

- O sistema de tratamento apresentou evapotranspiração entre 4,9 e $18,7 \mathrm{~mm} \mathrm{~d}^{-1}$, no período avaliado, correspondendo a uma perda de água entre $11 \%$ e $27 \%$ da vazão afluente;

- O coeficiente da cultura (Kc) da taboa variou de acordo com o estágio fenológico da planta, situando-se entre 2,22 e 4,58. Os maiores valores foram obtidos na fase de florescimento e o mais baixo logo após o corte das plantas de taboa; e

- A significativa perda de água por evapotranspiração nos SACs deve ser considerada no dimensionamento dos mesmos. Ademais, a avaliação das eficiências de remoção dos poluentes deve ser feita com base na carga removida, e não na concentração removida.

\section{REFERÊNCIAS}

ALLEN, R. G.et al. Crop evapotranspiration: guidelines for computing crop water requirements. Rome: FAO, 301p. Irrigation and Drainage Paper, 56. 1998.

BORIN, M. et al. Seasonal effects on residence time distribution and on water and chemical budgets in a phytotreatment pilot-plant. Proceedings: $6^{\text {th }}$ International Conference on Waste Stabilisation Ponds and 9th International Conference on Wetland Systems for Water Pollution Control. Avignon, France, IWA/Astee, OC-30, CD-ROM. $26^{\text {th }}$ of OC-30. Sept. - $1^{\text {st }}$ of October $/ 2004$
BRASIL, M. S. et al. Qualidade do efluente de sistemas alagados construídos, utilizados no tratamento de esgoto doméstico. Revista Brasileira de Engenharia Agrícola e Ambiental, v.9, (suplemento), p.133-137, 2005.

BRASIL, M. S.et al. Modeling of pollution removal in constructed wetlands with horizontal subsurface flow. Agrartechnische Forschung, v.13, n.2, p.48-56, 2007.

BRIX, H. Do macrophytes play a role in constructed treatment wetlands? Water Science Technology, v.35, n.5, p.11-17, 1997.

CADELLI, D. et al. Infuence of evapotranspiration on the design of extensive wastewater treatment systems under Mediterranean conditions at the MHEA ${ }^{\circledR}$ Experimental Centre of M Diq (Tetouan Moroco). Proceedings: $6^{\text {th }}$ International Conference on Waste Stabilisation Ponds and 9th International Conference on Wetland Systems for Water Pollution Control. Avignon, France, IWA/Astee, p. $93-103.26^{\text {th }}$ of Sept. - $1^{\text {st }}$ of October $/ 2004$.

DAVIS, L. A. Handbook of Constructed Wetlands. A Guide to Creating Wetlands for: Agricultural Wastewater, Domestic Wastewater, Coal Mine Drainage, Stormwater in the Mid-Atlantic Region, Volume 1: (USEPA Region III with USDA, NRCS, ISBN 0-16-052999-9), 1995.

FERREIRA, P. A. Curso de Engenharia Agrícola - Drenagem de terras agrícolas. Viçosa: UFV/DEA, 211 p. 2002.

ITRC-INTERSTATE TECHNOLOGY \& REGULATORY COUNCIL.Technical and Regulatory GuidanceDocument for Constructed Treatment Wetlands. New Jersey: ITRC, 199p. December/2003.

LIM, P. E.; WONG, T. F.; LIM, D. V. Oxygen demand, nitrogen and copper removal by free-water-surface and subsurface-flow constructed wetlands under tropical conditions. Environment International, v. 26, n. 5-6, p.425-431. May/2001.
REED, S, C.; CRITES, R. W.; MIDDLEBROOKS, E. J. Natural systems for management and treatment. New York: McGraw-Hill, Inc. 435p. 1995.

SÁNCHEZ-CARRILLO, S.; ÁLVAREZCOBELAS, M; BENITEZ, M. A simple method for estimating water loss by transpiration in wetlands. Hydrological Sciences-Journal-des Hydrologiques. v.46, n.4, p. 537-552. August/2001.

SANFORD, W. E. et al. Hydraulic conductivity of gravel and sand as substrates in rock-reed filters. Ecological Engineering, v.4, p.321-336, 1995.

SOUSA, J. T.et al. Utilização de wetland construido no pós-tratamento de esgotos domésticos pré-tratados em reatores UASB. Engenharia Sanitária e Ambiental, v.9, n.4, p.285-290. Out/Dez, 2004.

TANNER, C. C.; \& SUKIAS, J. P. Accumulation of organic solids in gravel-bed constructed wetlands. Water Science Technology, v.32, n.3, p.229-239, 1995.

U.S. Environmental Protection Agency. Manual Constructed Wetlands for Municipal Wastewater Treatment. EPA 625-R-99-010, US EPA ORD 165 p. Cicinnati: Ohio, 2000 b.

Endereço para correspondência:

Mozart da Silva Brasil

Rodovia 399, km 05, Zona Rural

C. Postal 47

78996-000 Colorado do Oeste

- RO - Brasil

Tel.: (69) 334I-7805

E-mail: mozart-brasil@bol.com.br 